\section{Brain Stimulation for Basal Ganglia Disorders}

\section{Connie Pidgeon ${ }^{1}$ and Andrea Eugenio Cavanna ${ }^{1,2 *}$}

The basal ganglia (BG) have long been considered a purely motor region of the brain. However, over the last couple of decades it has progressively become clear that BG are central components of different cortical-subcortical circuits, described on a basic level as 'motor', 'oculomotor', 'associative' and 'limbic', according to the function of the cortical regions involved [1]. In turn, each circuit encompasses multiple, functionally and anatomically distinct subcircuits. Dysfunction within these segregated circuits has been shown to cause BG disorders. Specifically, Parkinson disease (PD) and dystonia, are thought to arise from disturbances within the BGthalamo-cortical motor circuitry, whilst Tourette syndrome (TS) and obsessive-compulsive disorder (OCD), seem to be related to abnormalities within the limbic circuitry [2]. There is also a degree of overlap between the motor and behavioural sides of BG disorders, as conditions like PD and TS are characterised by both movement disorders and neuropsychiatric symptoms [3].

The limitations in terms of both efficacy and tolerability of medications and other therapeutic modalities used to treat BG disorders, alongside the progression in the understanding of the pathophysiological basis of BG disorders, has led to a rekindled interest in functional neurosurgery for the treatment of these conditions, particularly the use of deep brain stimulation (DBS). DBS therapy involves chronic electrical stimulation of specific subcortical areas, selectively modifying activity at the level of specific targets, whilst avoiding uninvolved circuits and thus widespread adverse affects. In BG disorders, traditional ablative neurosurgical procedures have largely been superseded by DBS due to its less invasive, reversible and modifiable nature [4].

DBS has been shown to be a highly effective and generally safe treatment strategy for a variety of BG disorders $[5,6]$. Following its introduction in the 1990s, DBS is now commonly used in patients with advanced $\mathrm{PD}$, especially in the presence of persistent severe tremor or disabling drug-induced complications. Stimulation of different brain areas produces different clinical effects in patients with $\mathrm{PD}$, with the subthalamic nucleus (STN) and the internal portion of the globus pallidus (GPi) being the most commonly targeted regions [7].

DBS of the BG is also being investigated for severe, refractory neuropsychiatric disorders, although its use for the treatment of these conditions is at an earlier stage compared to PD. Over the past

\footnotetext{
*Corresponding author: Dr. Andrea Eugenio Cavanna MD PhD, Department of Neuropsychiatry, The Barberry National Centre for Mental Health, Birmingham B15 2FG, United Kingdom, Tel +44 121 3012317; Fax +44 121 3012291; E-mail: a.cavanna@ion.ucl.ac.uk
}

Received: June 16, 2012 Accepted: June 18, 2012 Published: June 20, 2012 decade, the use of DBS for patients with severe, treatment-resistant TS has been investigated with increasing attention. Although initial findings are encouraging mainly in terms of tic severity outcomes, the majority of research is limited to case reports or small open-label trials [6]. A larger 18-patient study showed that all patients responded well to DBS [8], with no adverse effects reported. A follow up study at 2 years also reported a $53 \%$ improvement in tic severity, as well as continued improvements in other co-morbid symptoms and health-related quality of life [9]. A recent double-blind crossover trial demonstrated that thalamic DBS improved tic severity, despite a high incidence of adverse effects, including low energy in all patients [10]. Interpretation of results on this topic is complicated by the symptomatic heterogeneity across patients, differences in BDS targets (different components of the fronto-striato-thalamo-cortical loop) and outcome measures. The lack of adequate study design, including low statistical power and uncontrolled variables, further complicates the interpretation of these early findings. Consistently, a recent review concluded that at this stage it is not possible to identify optimal targets for DBS (BG/thalamus) in patients with TS due to the variability across studies in target confirmation, symptomatic outcome measures used and follow-up duration [11].

Results from several case reports and small case series indicate that DBS targeting the anterior limb of the IC in patients with OCD produces sustained symptomatic improvements, with few reported adverse effects [7]. More recently, alternative brain areas have been targeted for the treatment of OCD. A large double-blind crossover trial involving 16 patients showed that OCD severity was significantly lower after DBS targeting the limbic-associative area of the STN than after sham surgery [12]. Preliminary research has also been undertaken investigating the effects of DBS in refractory depression (targeting limbic BG areas) and addiction (targeting the nucleus accumbens, a critical structure of the reward system), with overall positive results [6].

In conclusion, DBS has been shown to be an effective treatment for BG disorders affecting movement. Preliminary research has hinted at its potential for the treatment of severe, refractory neuropsychiatric disorders, although the evidence is not yet conclusive in this emerging field. A possible reason for the relative lack of research in this area may be that the DBS targets complicated and multifaceted symptoms in neuropsychiatric disorders, in contrast to the relatively straightforward and easily measurable symptoms in movement disorders. The vulnerability of patients with severe psychiatric disorders and the potential modification of personality that neurosurgery involves also raises pertinent ethical issues surrounding the use of DBS in neuropsychiatric conditions [6]. Future research is clearly warranted to determine the best anatomical targets and exact mechanism of action, to construct a robust evidence base for its indication and - perhaps most importantly - to determine the effects on patient's health-related quality of life. International collaborations in large, multicentre randomised controlled trials are likely to be central for the development of this fascinating field within functional neurosurgery. 


\section{References}

1. Alexander GE, DeLong MR, Strick PL (1986) Parallel organization of functionally segregated circuits linking basal ganglia and cortex. Annu Rev Neurosci 9: 357-381.

2. Hoover JE, Strick PL (1993) Multiple output channels in the basal ganglia. Science 259: 819-821.

3. Agid Y, Arnulf I, Bejjani P, Bloch F, Bonnet AM, et al. (2003) Parkinson's disease is a neuropsychiatric disorder. Adv Neurol 91: 365-370.

4. Wichmann T, DeLong MR (2006) Deep brain stimulation for neurologic and neuropsychiatric disorders. Neuron 52: 197-204.

5. Welter ML, Grabli D, Vidailhet M (2010) Deep brain stimulation for hyperkinetics disorders: dystonia, tardive dyskinesia, and tics. Curr Opin Neurol 23: 420-425.

6. Krack P, Hariz Ml, Baunez C, Guridi J, Obeso JA. (2010) Deep brain stimulation: from neurology to psychiatry? Trends Neurosci 33: 474-484.

7. Perlmutter JS, Mink JW (2006) Deep brain stimulation. Annu Rev Neurosci 29: $229-257$
8. Servello D, Porta M, Sassi M, Brambilla A, Robertson MM (2008) Deep brain stimulation in 18 patients with severe Gilles de la Tourette syndrome refractory to treatment: the surgery and stimulation. J Neurol Neurosurg Psychiatry 79: 136-142.

9. Porta M, Brambilla A, Cavanna AE, Servello D, Sassi M, et al. (2009) Thalamic deep brain stimulation for treatment-refractory Tourette syndrome: two-year outcome. Neurology 73: 1375-1380.

10. Ackermans L, Duits A, van der Linden C, Tijssen M, Schruers K, et al. (2011) Double-blind clinical trial of thalamic stimulation in patients with Tourette syndrome. Brain 134: 832-844.

11. Pansaon Piedad JC, Rickards HE, Cavanna AE (2012) What patients with Gilles de la Tourette Syndrome should be treated with deep brain stimulation and what is the best target? Neurosurgery 71:173-192.

12. Mallet L, Polosan M, Jaafari N, Baup N, Welter ML, et al. (2008) Subthalamic nucleus stimulation in severe obsessive-compulsive disorder. N Engl J Med 359: 2121-2134.

\section{Author Affiliations}

${ }^{1}$ The Michael Trimble Neuropsychiatry Research Group, Department of Neuropsychiatry, BSMHFT and University of Birmingham, United Kingdom ${ }^{2}$ Sobell Department of Motor Neuroscience and Movement Disorders, Institute of Neurology and University College London, United Kingdom

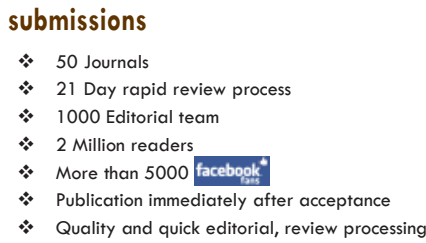
submissions

* 50 Journals

* 21 Day rapid review process

1000 Editorial tean

* 2 Million readers

- More than 5000 facebogk

- Publication immediately after acceptance

* Quality and quick editorial, review processing

Submit your next manuscript and get advantages of SciTechno

Submit your next manuscript at $\bullet$ www.scitechnol.com/submission 